\title{
Integration of Social Behavioral Modeling for Energy Optimization in Smart Environments
}

\author{
Simone Silvestri, D. A. Baker, Valeria Dolce \\ Missouri University of Science and Technology \\ silvestris@mst.edu,bakerden@mst.edu,dolcev@mst.edu
}

\begin{abstract}
A key requirement for success of smart home energy management systems is understanding the user's psychological perception of a smart environments, and the design of control strategies that specifically take into account such dimensions in system operation. We discuss how our research develops psychological models and integrates them with optimization and machine learning techniques to realize social and behavioral aware energy optimization methodologies for smart homes.
\end{abstract}

\section{CCS CONCEPTS}

•Applied computing $\rightarrow$ Psychology; •Computer systems organization $\rightarrow$ Embedded software;

\section{KEYWORDS}

Smart Living, Social-behavioral models, Energy management.

\section{ACM Reference format:}

Simone Silvestri, D. A. Baker, Valeria Dolce. 2016. Integration of Social Behavioral Modeling for Energy Optimization in Smart Environments. In Proceedings of SocialSens'17,, Pittsburgh, PA, USA, April 21 2017, 1 pages. DOI: http://dx.doi.org/10.1145/3055601.3055618

\section{INTRODUCTION}

A key building block for energy management of future smart cities are intelligent residential environments, generally termed smart homes. These homes will include a plethora of smart interconnected appliances, realized through the Internet of Things (IoT) paradigm. This creates an opportunity to improve the energy efficiency of the house by monitoring and controlling the schedule of smart appliances [1,2]. However, enabling automatic energy management implicitly requires a loss of control for the user, which should not be neglected. Indeed, there are a number of psychological phenomena that could hijack a system that takes away too much user control, regardless of the potential benefits [3]. The integration of these psychological aspects in the design and operation of smart environments has been largely overlooked by previous approaches. As a result, previously proposed systems could be perceived as a threat to a user's autonomy, which could lead users to engage in wasteful energy consumption and/or to develop negative attitudes

Permission to make digital or hard copies of all or part of this work for personal or classroom use is granted without fee provided that copies are not made or distributed for profit or commercial advantage and that copies bear this notice and the full citation on the first page. Copyrights for components of this work owned by others than ACM must be honored. Abstracting with credit is permitted. To copy otherwise, or republish, to post on servers or to redistribute to lists, requires prior specific permission and/or a fee. Request permissions from permissions@acm.org.

SocialSens'17,, Pittsburgh, PA, USA

(C) 2017 ACM. 978-1-4503-4977-2/17/04 ..\$\$15.00

DOI: http://dx.doi.org/10.1145/3055601.3055618 that can ultimately lead to abandonment and avoidance of such systems.

Below we describe our innovative and interdisciplinary research framework that aims at realizing a social-behaviorally aware management system for energy optimization of smart environments.

\section{OVERVIEW OF THE PROPOSED RESEARCH}

Social behavioral user modeling for smart home environments. We initially design and administer a large scale, online, social behavioral (SB) survey to assess and model perceived value and importance of particular appliances, or groups of appliances, and how these perceptions change within different energy availability contexts. Importance is expressed in terms of five psychological dimensions of well-being. Further, SB models are derived within the context of demographic information such as household size and socioeconomic status.

Formal models of user behavior. We design formal models derived by experimental surveys to define concepts, such as user utility, using graph theory. Machine learning techniques are adopted to correlate SB dimensions to quantitative metrics observable by smart devices. On the basis of these correlations, we design algorithms that refine the user model and dynamically adapt to the specific needs of the inhabitants of the smart home.

Social-behavior-aware optimization of energy consumption. We consider the specific context of energy constrained environments. These constraints may be self-imposed by the user to reduce energy bills, or as a result of a contingency in the main power grid. We exploit the user models to design optimization problems and efficient online algorithms to schedule user appliances and meet the energy budget while minimizing impact on the user's lifestyle. Psychological and behavioral factors of efficacy and user engagement. We examine the interaction between key psychological factors related to persuasion and the interface design of the energy management system that facilitate or hinder user engagement and energy savings. This is achieved through a series of online experimental surveys and a longitudinal study in a real world settings.

\section{ACKNOWLEDGMENTS}

Supported by the National Institute for Food Agriculture grant 2017-67008-26145.

\section{REFERENCES}

[1] Pietro Cottone, Salvatore Gaglio, Giuseppe Lo Re, and Marco Ortolani. 2015. User activity recognition for energy saving in smart homes. Pervasive and Mobile Computing 16 (2015), 156-170.

[2] Debraj De, Shaojie Tang, Wen-Zhan Song, Diane Cook, and Sajal K Das. 2012. ActiSen: Activity-aware sensor network in smart environments. Pervasive and Mobile Computing 8, 5 (2012), 730-750.

[3] The Networking, Information Technology Research, and Development (NITRD). 2015 (accessed March 9, 2017). Cyber Physical Systems Vision Statement. 